\title{
Contribution of Hardening Mechanism to VVER-1000 RPV Welds Flux Effect
}

\section{E. A. Kuleshova ${ }^{1,2}$, B. A. Gurovich ${ }^{1}$, A. S. Frolov ${ }^{1}$, D. A. Maltsev' ${ }^{1}$, Z. V. Bukina ${ }^{1}$, G. M. Zhuchkov', and S. V. Fedotova ${ }^{1}$}

${ }^{1}$ National Research Center "Kurchatov Institute", Akademika Kurchatova pl. 1, Moscow, 123182, Russia

${ }^{2}$ National Research Nuclear University "MEPhl" (Moscow Engineering Physics Institute), Kashirskoe highway 31, Moscow, 115409, Russia

\section{Abstract}

Systematic differences in the radiation embrittlement kinetics of steels irradiated with different fluxes requires a clear understanding and assessment of the mechanisms responsible for the flux effect. This paper presents results of research of hardening mechanism contribution to flux effect of VVER-100o reactor pressure vessel (RPV)

Corresponding Author:

E. A. Kuleshova

evgenia-orm@yandex.ru

Received: 21 December 2017

Accepted: 15 April 2018

Published: 6 May 2018

Publishing services provided by Knowledge $\mathrm{E}$

(c) E. A. Kuleshova et al. This article is distributed under the terms of the Creative Commons Attribution License, which permits unrestricted use and redistribution provided that the original author and source are credited.

Selection and Peer-review under the responsibility of the MIE-2017 Conference Committee.

\section{G OPEN ACCESS}

\section{Introduction}

Radiation embrittlement of reactor pressure vessel (RPV) steels (increase of critical brittleness temperature $T_{K}$ ) is due to the action of hardening mechanism (radiationinduced changes in the phase composition of material) and the non-hardening one (formation of grain boundary and intergranular segregations). The most reliable predictions of the mechanical properties degradation of RPV material are based on the surveillance specimens (SS) studies that are under long term exposure to operational factors typical to RPV operating conditions [1-5].

The fluence accumulation rate (flux) for the SS is the closest one to the irradiation conditions of the RPV inner wall (leading factor of 0.5-3.0). However, an anticipatory prediction of materials radiation embrittlement degree requires an accelerated irradiation of specimens in research reactors to fluence values close and beyond the designed ones. The leading factor of irradiation in research reactors in comparison with the RPV inner wall irradiation in the core area can reach $\sim 30-200$ times. 
It has been shown previously [6] that RPV weld metal (WM) with high nickel content have a slightly lower density and size of precipitates under accelerated irradiation. The lower radiation embrittlement rate of WM was observed for the studied fast neutron fluences and fluxes ranges [7]. Flux effect was not observed for base metal (BM) [8].

More accurate assessment of the hardening mechanism contribution to flux effect observed in VVER-1000 weld metal with high nickel content under accelerated irradiation requires detailed comparative investigations using transmission electron microscopy (TEM) and atom probe tomography (APT). It is possible by carrying out a comparative analysis of SS and temperature set of SS subsequently accelerated irradiated in a research reactor IR-8 to the SS weld metal fluence.

\section{Materials and methods}

Assessment of the hardening mechanism contribution to flux effect of VVER-1000 RPV weld metal was carried out on example high nickel content samples (1,73 wt \% Ni). The radiation set of SS as well as the temperature set of SS subsequently accelerated irradiated in a research reactor IR-8 to the radiation set fluence were used.

The chemical composition of the material is presented in Table 1. Irradiation parameters of the samples are presented in Table 2.

TABLE 1: Chemical composition of the studied VVER-1000 RPV weld metal (in wt. \%).

\begin{tabular}{|c|c|c|c|c|c|c|c|c|c|c|}
\hline \multirow[t]{2}{*}{ Material } & \multicolumn{10}{|c|}{ The elemental composition, mass. $\%$} \\
\hline & C & $\mathrm{Ni}$ & $P$ & $\mathrm{Cu}$ & S & $M n$ & $\mathrm{Si}$ & $\mathrm{Cr}$ & Mo & V \\
\hline Sv-10KhGNMAA & 0.07 & 1.73 & 0.008 & 0.03 & 0.012 & 0.98 & 0.30 & 1.72 & 0.63 & 0.02 \\
\hline
\end{tabular}

TABLE 2: The investigated states of the VVER-1000 weld metal studied samples $[6,8]$

\begin{tabular}{|c|c|c|c|}
\hline State & $\begin{array}{c}\text { Fluence (F), } \\
10^{22} \mathrm{~m}^{-2}\end{array}$ & $\begin{array}{l}\text { Flux, } 10^{14} \\
\mathrm{~m}^{-2} \mathrm{~s}^{-1}\end{array}$ & Time, $10^{3} \mathrm{~h}$ \\
\hline Irradiation within SS 3 & 45.4 & 12.5 & 125 \\
\hline $\begin{array}{l}\text { 3d temperature set }+ \\
\text { accelerated irradiation in } \\
\text { IR-8 }\end{array}$ & $45 \cdot 5$ & 118 & $\begin{array}{l}125 \text { (thermal aging) } \\
0.8 \text { (accelerated } \\
\text { irradiation) }\end{array}$ \\
\hline
\end{tabular}

The density and size of radiation-induced elements responsible for radiation hardening were determined by TEM (radiation defects - dislocation loops) and APT (Ni reached precipitates). TEM investigations were performed using Titan 80-300 (FEl, USA). APT investigations were performed using LEAP-4000 HR (Cameca, France). 


\section{Results}

Table 3 presents the results of comparative TEM and APT analysis (density, size and the average composition of the radiation-induced structural elements) of WM samples which were irradiated within SS as well as accelerated irradiated in research reactor IR8 (temperature set + accelerated irradiation). Figure 1 presents darkfield TEM-images of dislocation loops in the investigated samples. Figure 2 presents 3D APT-maps of the alloying elements in the investigated samples.

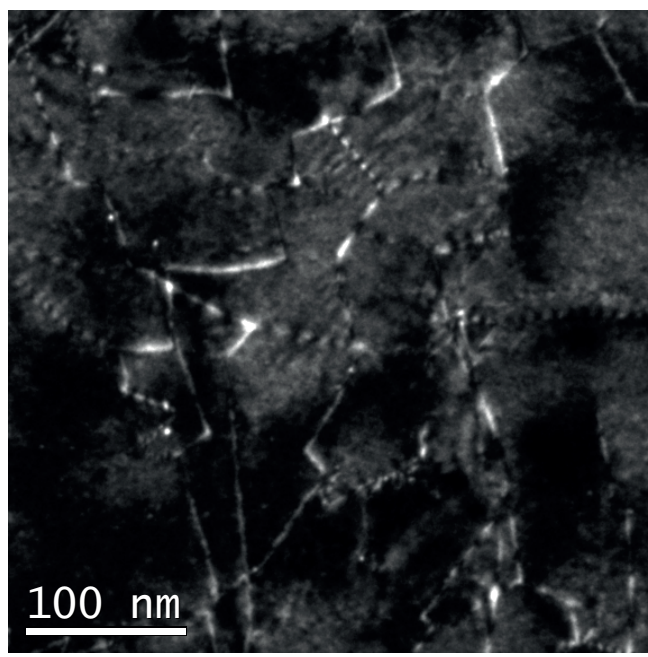

a - irradiation within SS

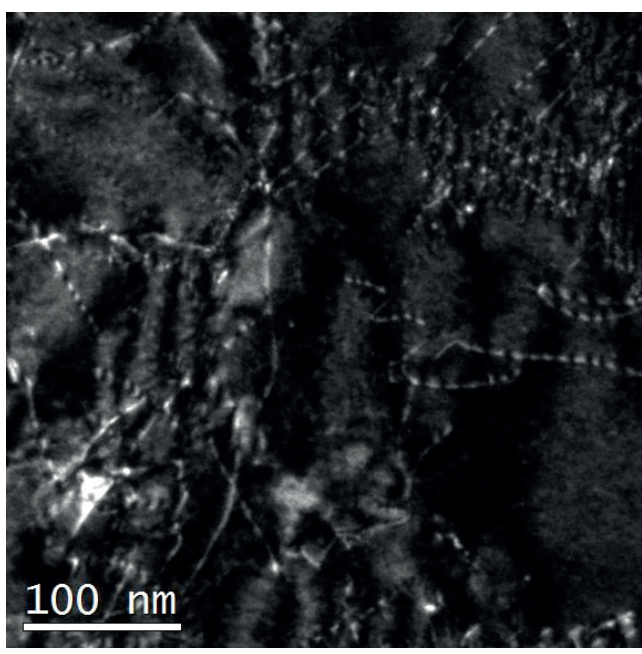

$\mathrm{b}$ - temperature set + accelerated irradiation

Figure 1: Darkfield TEM-images of dislocation loops in the investigated samples.

TABLE 3: The density, size and averaged composition of radiation-induced structural elements in VVER1000 RPV WM irradiated with different fluxes.

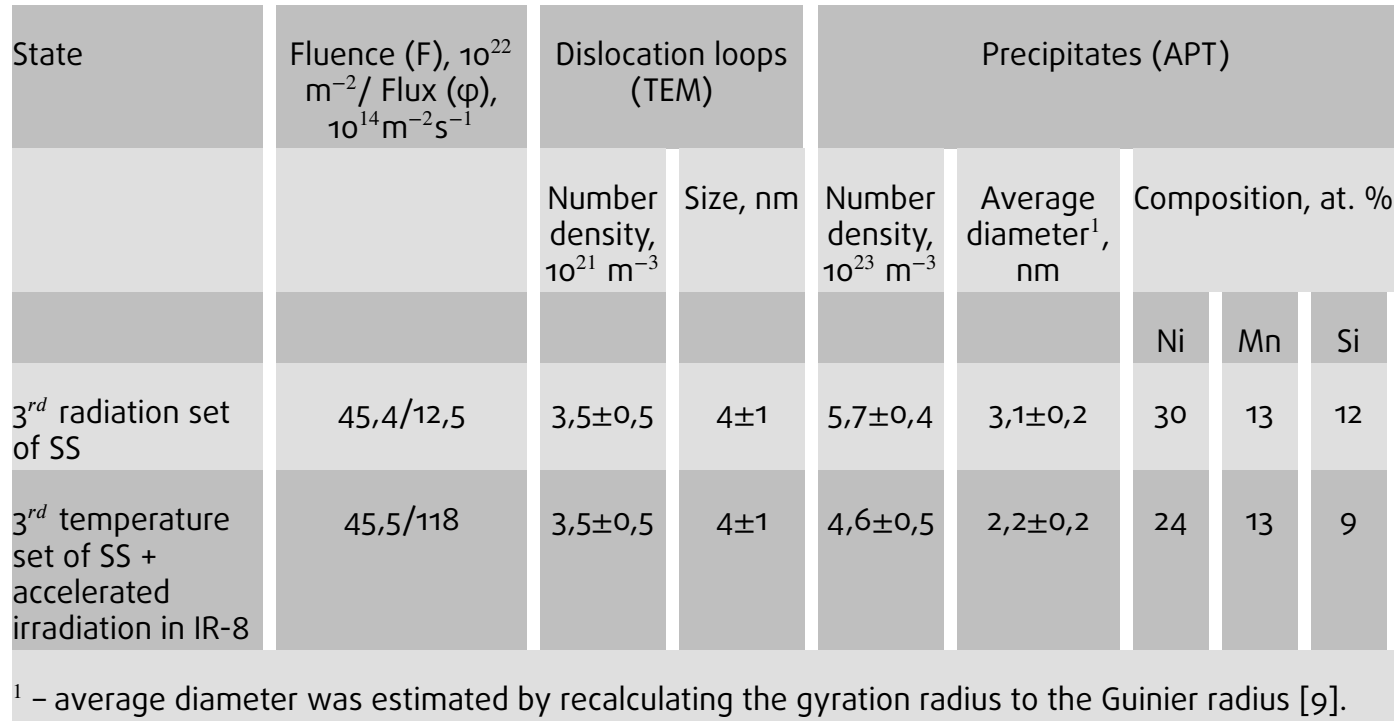



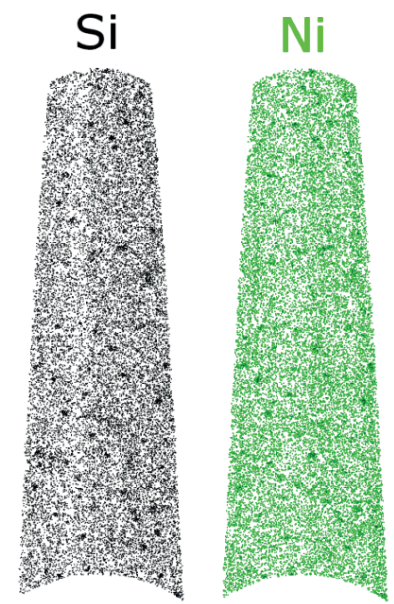

$40 \mathrm{~nm}$

a - irradiation within SS
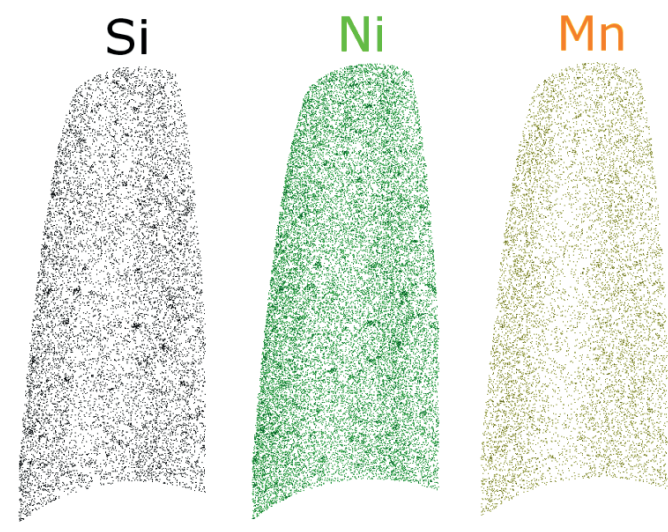

$40 \mathrm{~nm}$

Figure 2: 3D APT-maps of the alloying elements in the investigated samples.

\section{Results and discussion}

Since there is no change in the density and sizes of VVER-1000 materials strengthening phases (carbides mainly) $[6,10]$ decisive contribution to the hardening is due to formation dislocation loops and radiation-induced precipitates. It is possible to assess the yield strength change due to formation of radiation-induced structural elements using the Orowan equation [11]:

$$
\Delta \sigma_{0,2}=\alpha \cdot M \cdot G \cdot b \cdot \sqrt{\rho \cdot d}
$$

where $M$ is the Taylor factor, $G$ is the Young modulus and $b$ is the Burgers vector module; $\rho$ and $d$ are experimentally determined density of defects and its average size respectively. The strength of the barrier is expressed by the constant $\alpha$.

Considering the contribution of different types of structural elements in the radiation hardening additive, we get the following relation:

$$
\Delta \sigma_{0,2}=\Delta \sigma_{\text {prec }}+\Delta \sigma_{\text {loops }}
$$

For the specimens after acceleration irradiation $\left(\Delta \sigma_{0,2}^{\text {accel }}\right)$ and SS $\left(\Delta \sigma_{0,2}^{\text {SS }}\right)$ the following ratio of the yield stress shifts can be obtained:

$$
\frac{\Delta \sigma_{0,2}^{\text {accel }}}{\Delta \sigma_{0,2}^{\mathrm{SS}}}=\frac{\Delta \sigma_{\text {prec }}^{\text {accel }}+\Delta \sigma_{\text {loops }}^{\text {accel }}}{\Delta \sigma_{\text {prec }}^{\mathrm{SS}}+\Delta \sigma_{\text {loops }}^{\mathrm{SS}}}
$$


where $\Delta \sigma_{\text {prec }}^{\text {acc }} \Delta \sigma_{\text {loops }}^{\text {acc }}$ - yield strength shift contribution due to the formation of radiation-induced precipitates and dislocation loops for the accelerated irradiation; $\Delta \sigma_{\text {prec, }}^{\text {SS }} \Delta \sigma_{\text {loops }}^{\text {SS }}$ - yield strength shift contribution due to the formation of radiationinduced precipitates and dislocation loops for the irradiation within SS;

Since the dislocation loops densities for accelerated irradiation and irradiation within SS are the same (as shown in Table 3) $\Delta \sigma_{\text {loops }}^{\text {SS }}=\Delta \sigma_{\text {loops }}^{\text {acc }}=\Delta \sigma_{\text {loops' }}$ the expression (3) takes form:

$$
\begin{aligned}
\frac{\Delta \sigma_{0,2}^{\mathrm{acc}}}{\Delta \sigma_{\mathrm{o}, 2}^{\mathrm{SS}}}= & \frac{\Delta \sigma_{\mathrm{prec}}^{\mathrm{acc}}+\Delta \sigma_{\text {loops }}}{\Delta \sigma_{\mathrm{prec}}^{\mathrm{SS}}+\Delta \sigma_{\text {loops }}}=\frac{\Delta \sigma_{\mathrm{prec}}^{\mathrm{acc}} / \Delta \sigma_{\text {loops }}+1}{\Delta \sigma_{\mathrm{prec}}^{\mathrm{SS}} / \Delta \sigma_{\text {loops }}+1} \frac{\Delta \sigma_{0,2}^{\mathrm{acc}}}{\Delta \sigma_{\mathrm{o}, 2}^{\mathrm{SS}}} \\
= & \frac{\Delta \sigma_{\mathrm{prec}}^{\mathrm{acc}}+\Delta \sigma_{\text {loops }}}{\Delta \sigma_{\mathrm{prec}}^{\mathrm{SS}}+\Delta \sigma_{\text {loops }}}=\frac{\Delta \sigma_{\mathrm{prec}}^{\mathrm{acc}} / \Delta \sigma_{\text {loops }}+1}{\Delta \sigma_{\mathrm{prec}}^{\mathrm{SS}} / \Delta \sigma_{\text {loops }}+1}
\end{aligned}
$$

Substituting (1) into (4) we obtain:

$$
\begin{aligned}
& \frac{\Delta \sigma_{0,2}^{\mathrm{acc}}}{\Delta \sigma_{\mathrm{o}, 2}^{\mathrm{sS}}}=\frac{\alpha_{\text {prec }} \cdot \sqrt{\rho_{\mathrm{prec}}^{\mathrm{acc}} \cdot \mathrm{d}_{\mathrm{prec}}^{\mathrm{acc}}} / \alpha_{\text {loops }} \cdot \sqrt{\rho_{\text {loops }} \cdot \mathrm{d}_{\text {loops }}}+1}{\alpha_{\text {prec }} \cdot \sqrt{\rho_{\mathrm{prec}}^{\mathrm{sS}} \cdot \mathrm{d}_{\mathrm{prec}}^{\mathrm{sS}}} / \alpha_{\text {loops }} \cdot \sqrt{\rho_{\text {loops }} \cdot \mathrm{d}_{\text {loops }}}+1} \frac{\Delta \sigma_{0,2}^{\mathrm{acc}}}{\Delta \sigma_{0,2}^{\mathrm{sS}}} \\
& =\frac{\alpha_{\text {prec }} \cdot \sqrt{\rho_{\text {prec }}^{\text {acc }} \cdot \mathrm{d}_{\text {prec }}^{\text {acc }}} / \alpha_{\text {loops }} \cdot \sqrt{\rho_{\text {loops }} \cdot \mathrm{d}_{\text {loops }}}+1}{\alpha_{\text {prec }} \cdot \sqrt{\rho_{\text {prec }}^{\mathrm{SS}} \cdot \mathrm{d}_{\text {prec }}^{\mathrm{SS}}} / \alpha_{\text {loops }} \cdot \sqrt{\rho_{\text {loops }} \cdot \mathrm{d}_{\text {loops }}}+1}
\end{aligned}
$$

Take into account that according to [11] $\alpha_{\text {loops }}=0,33$ and $\alpha_{\text {prec }}=0,08$ we obtain:

$$
\Delta \sigma_{0,2}^{\mathrm{SS}}=1,22 \cdot \Delta \sigma_{0,2}^{\mathrm{acc}}
$$

This result demonstrates that the hardening mechanism contribution to the flux effect is not crucial and confirms the previous NRC «Kurchatov Institute» works in which it was shown [2] that decisive influence of second radiation embrittlement mechanism on the flux effect - the formation of grain boundary segregations.

\section{Conclusion}

Studies of hardening phases of RPV-steel (VVER-1000) after accelerated irradiation carried out by TEM and APT using Orowan ratio allowed to estimate the contribution of the hardening mechanism to flux effect.

As a result, it was found that smaller size and density of the radiation-induced precipitates can not explain the decrease of radiation embrittlement rate in the case of accelerated irradiation compared with irradiation within SS. 
This is a proof of decisive influence of second radiation embrittlement mechanism on the flux effect - the formation of grain boundary segregations.

\section{References}

[1] Brumovsky M. Check of Master Curve application to embrittled RPVs of WWER type reactors. 2002. Vol. 79. P. 715-721.

[2] D. Erak, B. Gurovich, E. Kuleshova, Y. Shtrombakh, D. Zhurko, V. Papina, Radiation Embrittlement of VVER-1000 Reactor Pressure, in: 22nd Conf. Struct. Mech. React. Technol. San Fr. California, USA - August 18-23, 2013.

[3] Shtrombakh Y.I., Gurovich B.A., Kuleshova E.A., Erak D.Y., Fedotova S. V., Zhurko D.A., Zabusov 0.O., Nikolaev Y.A. Experimental assessment of the effectiveness of recovery annealing of VVER-1000 vessels // At. Energy. 2011. Vol. 109. P. 257-265.

[4] Kryukov A., Nanstad R.K., Brumovsky M. Common comparison of the irradiation embrittlement of WWER / PWR reactor pressure vessel steels // Nucl. Eng. Des. Elsevier B.V., 2014. Vol. 273. P. 175-180.

[5] Miller M.K., Russell K.F., Kocik J., Keilova E. Atom probe tomography of 15Kh2MFA Cr-Mo-V steel surveillance specimens // Micron. 2001. Vol. 32, № 8. P. 749-755.

[6] Gurovich B., Kuleshova E., Shtrombakh Y., Fedotova S., Maltsev D., Frolov A., Zabusov 0., Erak D., Zhurko D. Evolution of structure and properties of VVER-100o RPV steels under accelerated irradiation up to beyond design fluences // J. Nucl. Mater. 2015. Vol. 456. P. 23-32.

[7] Gurovich B., Kuleshova E., Fedotova S., Maltsev D., Zabusov O., Frolov A., et al. 2014 In: Fontevraud 8 - Contrib. Mater. Investig. Oper. Exp. to LWRs' Safety, Perform. Reliab. Fr. Avignon - 2014, Sept. 14-18.

[8] Gurovich B. et al. The effect of radiation-induced structural changes under accelerated irradiation on the behavior of water-cooled reactor pressure vessel steels // Key Eng. Mater. 2014. Vol. 592-593. P. 573-576.

[9] Michael K. Miller. Atom Probe Tomography: Analysis at the Atomic Level. Springer Science \& Business Media, 2012. 239 p.

[10] Shtrombakh Y.I., Gurovich B.A., Kuleshova E. A., Maltsev D.A., Fedotova S. V., Chernobaeva A. A. Thermal ageing mechanisms of VVER-1000 reactor pressure vessel steels // J. Nucl. Mater. Elsevier B.V., 2014. Vol. 452, № 1-3. P. 348-358.

[11] Lambrecht M., Meslin E., Malerba L., Hernández-Mayoral M., Bergner F., Pareige P., Radiguet B., Almazouzi A. On the correlation between irradiation-induced 
microstructural features and the hardening of reactor pressure vessel steels // J. Nucl. Mater. Elsevier B.V., 2010. Vol. 406, № 1. P. 84-89. 